\title{
NITROGÊNIO E MOLIBDÊNIO NA ADUBAÇÃO DO FEIJOEIRO IRRIGADO'
}

\author{
ELI ANTONIO FULLIN, MOEMA BACHOUR ZANGRANDE, JOSÉ ANTÔNIO LANI, \\ LUCIANO FURTADO DE MENDONÇA ${ }^{2}$ e NILTON DESSAUNE FILHO ${ }^{3}$
}

\begin{abstract}
RESUMO - Procurou-se avaliar o efeito da aplicação do nitrogênio $(\mathrm{N})$ associado ou não à aplicação do molibdênio (Mo) no feijoeiro, cultivar Goytacazes, sob irrigação, em Latossolo Vermelho-Amarelo coeso distrófico de Linhares, ES. Os tratamentos foram dispostos em blocos ao acaso, com três repetições. As doses de $\mathrm{N}$ como uréia, foram de 0 e $10 \mathrm{~kg} / \mathrm{ha}$ no plantio e 0 e $40 \mathrm{~kg} / \mathrm{ha}$ em cobertura. No Mo como molibdato de amônio, foram aplicados, 0 e $20 \mathrm{~g} /$ ha na peletização das sementes, e 0 e $20 \mathrm{~g} / \mathrm{ha}$ via foliar. Um tratamento adicional constou da aplicação de $10 \mathrm{~kg} / \mathrm{h}$ a de $\mathrm{Ne} 40 \mathrm{~kg} / \mathrm{ha}$ de FTE BR 9 no plantio e $40 \mathrm{~kg} / \mathrm{ha}$ de $\mathrm{N}$ em cobertura. A maior produtividade de grãos foi obtida com a aplicação de $10 \mathrm{~kg} / \mathrm{ha}$ e $40 \mathrm{~kg} / \mathrm{ha}$ de $\mathrm{N}$ no plantio e em cobertura, respectivamente, associados a $20 \mathrm{~g} / \mathrm{ha}$ de Mo via foliar.
\end{abstract}

Termos para indexação: Phaseolus vulgaris, solo, irrigação, nutrientes.

\section{NITROGEN AND MOLYBDENUM FERTILIZATION IN DRY BEAN UNDER IRRIGATED CONDITIONS}

\begin{abstract}
This field experiment was conducted to evaluate the effect of application of nitrogen $(\mathrm{N})$ associated or not with molibdenum (Mo) application to the dry bean cultivar Goytacazes under irrigation. The soil was a cohesive Dark Yellow latosol (Oxisol) at Linhares, ES, Brazil. A randomized complete blocks design with three replications was used. Nitrogen rates as urea, ranged from 0 to $10 \mathrm{~kg} / \mathrm{ha}$ at planting and 0 to $40 \mathrm{~kg} / \mathrm{ha}$ sidressed. Molibdenum was mixed with the seeds and applied by foliar spraying at rates of 0 and $20 \mathrm{~kg} / \mathrm{ha}$ as ammonium molybdate. One additional treatment with $10 \mathrm{~kg} / \mathrm{ha}$ of $\mathrm{N}$ and $40 \mathrm{~kg} / \mathrm{ha}$ of FTE BR 9 at planting plus $40 \mathrm{~kg} / \mathrm{ha}$ of $\mathrm{N}$ sidressed was used. The highest grain yield was recorded with the application of $10 \mathrm{~kg} / \mathrm{ha}$ and $40 \mathrm{~kg} / \mathrm{ha}$ of $\mathrm{N}$ at planting and sidressed, respectively, associated to $20 \mathrm{~kg} / \mathrm{ha}$ of Mo by foliar spraying.
\end{abstract}

Index terms: Phaseolus vulgaris, soil, irrigation, nutrients.

\section{INTRODUÇÃO}

A cultura do feijoeiro foi instalada na área de ocorrência dos solos do terciário no Norte do Estado do Espírito Santo, graças aos incentivos governamentais da década de 80, passando a ocupar lugar de destaque quanto à área plantada e quanto ao

\footnotetext{
${ }^{1}$ Aceito para publicação em 3 de setembro de 1998.

${ }^{2}$ Eng. Agr., M.Sc., EMCAPA - Centro de Pesquisa e Desenvolvimento Norte, Caixa Postal 62, CEP 29900-970 Linhares, ES. E-mail: cpdn@escelsa.com.br

${ }^{3}$ Eng. Agr., M.Sc., EMCAPA, Caixa Postal 391, CEP 29001-970 Vitória, ES. E-mail: emcapa01@npd.ufes.br
}

retorno econômico proporcionado. No entanto, os cultivos sucessivos da cultura levaram a uma estagnação e redução da produtividade, com implicações desfavoráveis tanto sociais como econômicas.

Como a produtividade é dependente do genótipo e do ambiente, o uso adequado de fertilizantes é indispensável para que a cultura possa expressar todo o potencial produtivo, principalmente considerando a baixa fertilidade natural dos solos cultivados na região e a importância do fornecimento de nitrogênio e molibdênio para o feijoeiro. Nesse aspecto, o nitrogênio é um dos nutrientes que proporcionam maior número de respostas à cultura (Vieira, 1983). Elevação na produção de grãos também é obtida com 
o fornecimento do molibdênio, seja em aplicação no solo ou nas sementes (Braga, 1972; Junqueira Neto et al., 1977; Santos et al., 1979), como também em aplicação via foliar (Vieira et al., 1992; Amane et al., 1994; Vieira, 1994).

Este trabalho teve como objetivo avaliar os efeitos da aplicação de nitrogênio e molibdênio no feijoeiro irrigado.

\section{MATERIAL E MÉTODOS}

O experimento foi conduzido em condições de campo, na fazenda experimental de Sooretama, da EMCAPA, em Linhares, ES, com altitude média de $70 \mathrm{~m}$ e coordenadas geográficas de $19^{\circ} 07^{\prime}$ de latitude sul e $40^{\circ} 05^{\prime}$ de longitude oeste. O relevo predominante é plano, com solo classificado como Latossolo Vermelho-Amarelo coeso distrófico (Embrapa, 1978), com as seguintes características químicas (Defelipo \& Ribeiro, 1981) e físicas (Embrapa, 1979): $\mathrm{P}=28 \mathrm{mg} / \mathrm{dm}^{3} ; \mathrm{K}=2,02 \mathrm{mmol}_{\mathrm{c}} / \mathrm{dm}^{3}$; $\mathrm{Ca}=12,0 \mathrm{mmol}_{\mathrm{c}} / \mathrm{dm}^{3} ; \mathrm{Mg}=9,0 \mathrm{mmol}_{\mathrm{c}} / \mathrm{dm}^{3} ;$ $\mathrm{Al}=5,0 \mathrm{mmol}_{\mathrm{c}} / \mathrm{dm}^{3} ; \mathrm{H}+\mathrm{Al}=36,0 \mathrm{mmol}_{\mathrm{c}} / \mathrm{dm}^{3}$; $\mathrm{pH}\left(\mathrm{H}_{2} \mathrm{O}\right)=4,8$; matéria orgânica $=22,0 \mathrm{~g} / \mathrm{dm}^{3}$; CTC a pH 7,0 $=59,0 \mathrm{mmol}_{\mathrm{c}} / \mathrm{dm}^{3} ; \mathrm{V}=39 \%$; argila $=220 \mathrm{~g} / \mathrm{kg}$; silte $=10 \mathrm{~g} / \mathrm{kg}$; areia fina $=170 \mathrm{~g} / \mathrm{kg}$ e areia grossa $=600 \mathrm{~g} / \mathrm{kg}$.

O calcário dolomítico foi aplicado para elevar a saturação de bases a 70\%, distribuído a lanço e incorporado ao solo, cerca de sessenta dias antes do plantio.

Os tratamentos com variações de doses de $\mathrm{N}$ no plantio $(0$ e $10 \mathrm{~kg} / \mathrm{ha}$ de $\mathrm{N})$ e em cobertura $(0$ e $40 \mathrm{~kg} / \mathrm{ha}$ de $\mathrm{N})$, como uréia e doses de Mo no plantio ( 0 e 20 g/ha de Mo) e em cobertura ( 0 e $20 \mathrm{~g} / \mathrm{ha}$ de Mo), como molibdato de amônio, foram dispostos em delineamento de blocos ao acaso, com três repetições. Um tratamento adicional constou da aplicação de $10 \mathrm{~kg} / \mathrm{ha}$ de $\mathrm{N}$ e $40 \mathrm{~kg} / \mathrm{ha}$ de FTE BR 9 em pó no plantio e $40 \mathrm{~kg} / \mathrm{ha}$ de $\mathrm{N}$ em cobertura. O FTE BR 9 foi aplicado como fonte de Mo. Além das adubações conforme os tratamentos, foram aplicados no plantio $50 \mathrm{~kg} / \mathrm{ha}$ de $\mathrm{P}_{2} \mathrm{O}_{5}$ como superfosfato simples, e $30 \mathrm{~kg} /$ ha de $\mathrm{K}_{2} \mathrm{O}$ como cloreto de potássio.

A parcela experimental foi formada por cinco fileiras de $5 \mathrm{~m}$, num total de $12,5 \mathrm{~m}^{2}$, considerando como área útil as três fileiras centrais, eliminando-se $0,5 \mathrm{~m}$ em cada extremidade, perfazendo $6,0 \mathrm{~m}^{2}$.

O plantio foi realizado em abril de 1995, utilizando-se o feijão da cultivar Goytacazes, em espaçamento de 0,5 m entre linhas, com $10 \mathrm{~cm}$ entre plantas, deixando-se 10 a 12 plantas por metro linear após o desbaste. Durante a condução do experimento, foram efetuados os tratos culturais necessários. A irrigação foi por aspersão convencional, sendo a necessidade de água controlada por tensiômetros calibrados à tensão de 0,3 bar, com $10 \mathrm{~mm}$ de lâmina líquida por aplicação.

No início da floração foram coletadas, em cada parcela experimental, folhas amadurecidas para análise de $\mathrm{Ne}$ Mo (Malavolta et al., 1989), e plantas para avaliação de nodulação (Vidor et al., 1983). Em julho de 1995, efetuouse a colheita, avaliando-se as produções de grãos e extração de $\mathrm{N}$ e Mo pela parte aérea e grãos. A extração dos nutrientes foi realizada em uma subamostra de plantas $\left(0,4 \mathrm{~m}^{2}\right)$, dentro da área útil.

\section{RESULTADOS E DISCUSSÃO}

O número de nódulos, peso da matéria seca dos nódulos e o acúmulo de $\mathrm{N}$ na planta, no estádio de floração, nos diversos tratamentos, não apresentaram significância estatística a 5\% de probabilidade pelo teste de Duncan (Tabela 1). Os resultados indicaram alta população de rizóbios nativos. Estas estirpes não foram afetadas pela adição de $\mathrm{N}$ e Mo. A pequena dose de $\mathrm{N}$ no plantio não afetou o número e o peso da matéria seca dos nódulos, bem como o acúmulo de N na planta; no entanto, Siqueira \& Franco (1988) e Mercante et al. (1992) observaram que pequenas doses de $\mathrm{N}$ podem incrementar a nodulação, enquanto a alta disponibilidade de $\mathrm{N}$ não a favorece. Os altos índices de nodulação, com ou sem a adição de Mo, indicam, conforme Mercante et al. (1992), que a disponibilidade de Mo no solo pode ter sido aumentada com a calagem realizada.

Diferença estatística significativa foi observada na produção média de grãos, destacando-se o tratamento com $10 \mathrm{~kg} / \mathrm{ha}$ e $40 \mathrm{~kg} / \mathrm{ha}$ de $\mathrm{N}$ no plantio e em cobertura, respectivamente, associados a $20 \mathrm{~g} / \mathrm{ha}$ de Mo via foliar. Por outro lado, a aplicação de Mo na peletização das sementes e na ausência do $\mathrm{N}$ apresentou a menor produtividade $(1.924,9 \mathrm{~kg} / \mathrm{ha})$, o que representa uma redução de $20 \%$ na produção, em comparação com o tratamento mais produtivo.

A elevada produção da testemunha $(2.209,6 \mathrm{~kg} / \mathrm{ha})$ demonstra, em síntese, a alta fertilidade do solo quanto aos elementos estudados, favorecida, provavelmente, pela irrigação e pela eficiência das estirpes nativas de rizóbios na fixação de $\mathrm{N}_{2}$. 
TABELA 1. Nodulação e acúmulo de nitrogênio no estádio de floração e produtividade média de grãos do feijoeiro cultivar Goytacazes. Linhares, ES, 1995'.

\begin{tabular}{|c|c|c|c|c|c|c|c|}
\hline \multicolumn{4}{|c|}{ Tratamentos } & \multirow{3}{*}{$\begin{array}{l}\text { Número de } \\
\text { nódulos/ } \\
\text { planta }\end{array}$} & \multirow{3}{*}{$\begin{array}{l}\text { Peso da matéria } \\
\text { seca de nódulos } \\
\text { (mg/planta) }\end{array}$} & \multirow{3}{*}{$\begin{array}{l}\text { Acúmulo N } \\
\text { (mg/planta) }\end{array}$} & \multirow{3}{*}{$\begin{array}{l}\text { Produtividade } \\
\text { de grãos } \\
(\mathrm{kg} / \mathrm{ha})\end{array}$} \\
\hline \multicolumn{2}{|c|}{$\mathrm{N}(\mathrm{kg} / \mathrm{ha})$} & \multicolumn{2}{|c|}{ Mo (g/ha) } & & & & \\
\hline Plantio & Cobertura & Plantio & Cobertura & & & & \\
\hline 0 & 0 & 0 & 0 & $59,69 a$ & $82,27 \mathrm{a}$ & $308,12 \mathrm{a}$ & $2209,6 a b$ \\
\hline 10 & 0 & 0 & 0 & $39,19 a$ & $76,50 \mathrm{a}$ & $353,34 \mathrm{a}$ & $2147,6 \mathrm{abc}$ \\
\hline 0 & 40 & 0 & 0 & $25,18 \mathrm{a}$ & $53,80 \mathrm{a}$ & $349,79 a$ & $2185,5 \mathrm{abc}$ \\
\hline 10 & 40 & 0 & 0 & $44,73 \mathrm{a}$ & $84,47 a$ & $362,44 a$ & $2163,7 \mathrm{abc}$ \\
\hline 0 & 0 & 0 & 20 & $55,84 \mathrm{a}$ & $78,94 \mathrm{a}$ & $245,52 \mathrm{a}$ & $2043,7 \mathrm{abc}$ \\
\hline 10 & 0 & 0 & 20 & $38,87 \mathrm{a}$ & $43,57 \mathrm{a}$ & $313,47 a$ & $2119,4 \mathrm{abc}$ \\
\hline 0 & 40 & 0 & 20 & $62,93 \mathrm{a}$ & $72,08 \mathrm{a}$ & $339,16 a$ & $2231,3 \mathrm{ab}$ \\
\hline 10 & 40 & 0 & 20 & $45,99 \mathrm{a}$ & $66,15 \mathrm{a}$ & $284,07 \mathrm{a}$ & $2318,3 \mathrm{a}$ \\
\hline 10 & 0 & 20 & 0 & $47,55 \mathrm{a}$ & $78,03 \mathrm{a}$ & $223,67 a$ & $2194,1 \mathrm{abc}$ \\
\hline 0 & 40 & 20 & 0 & $36,66 a$ & $45,90 \mathrm{a}$ & $351,79 a$ & $2019,2 b c$ \\
\hline 10 & 40 & 20 & 0 & $35,69 \mathrm{a}$ & $56,23 a$ & $343,79 a$ & $2110,2 \mathrm{abc}$ \\
\hline 0 & 0 & 20 & 0 & $41,98 \mathrm{a}$ & $78,70 \mathrm{a}$ & $381,88 \mathrm{a}$ & $1924,9 \mathrm{c}$ \\
\hline 10 & 40 & $40^{2}$ & 0 & $39,64 \mathrm{a}$ & $48,37 \mathrm{a}$ & $373,23 \mathrm{a}$ & $2287,4 \mathrm{ab}$ \\
\hline C.V.(\%) & & & & 40,96 & 40,82 & 21,95 & 6,73 \\
\hline
\end{tabular}

${ }^{1}$ Médias seguidas da mesma letra nas colunas não diferem entre si (Duncan 5\%).

2 Tratamento adicional: $40 \mathrm{~kg} / \mathrm{ha}$ de FTE BR 9 aplicado no sulco de plantio.

Os teores médios de $\mathrm{N}$ nas folhas no estádio de floração (Tabela 2) variaram de $33,9 \mathrm{~g} / \mathrm{kg}$ a $41,9 \mathrm{~g} / \mathrm{kg}$, enquadrando-se no nível indicado por Malavolta et al. (1989) como adequado para o feijoeiro. As variações dos teores de $\mathrm{N}$ e $\mathrm{Mo}$ foliares foram estatisticamente significativas, sendo que, no geral, os maiores valores ocorreram para os tratamentos que receberam a adubação molíbdica via foliar, independentemente da aplicação do N. Na prática, isto significa que o fornecimento de Mo mediante a peletização das sementes não foi eficiente, e que o Mo comportou-se de forma semelhante à dos tratamentos em que o micronutriente não foi aplicado. Essa resposta diferenciada, segundo Berger et al. (1995), pode ser um comportamento intrínseco à cultivar utilizada. Esses autores verificaram, por exemplo, que na cultivar Ouro, o Mo pode ser aplicado com a mesma eficiência tanto nas sementes quanto na pulverização foliar, enquanto na cultivar Ouro Negro a pulverização foliar foi mais eficiente do que a peletização. Essas evidências permitem inferir que a cultivar Goytacazes, utilizada nesse estudo, comportou-se de modo semelhante à cultivar Ouro Negro. Amane et al. (1994), estudando o comportamento de 17 cultivares de feijão, já haviam constatado que a intensidade de resposta à aplicação de Mo pode variar consideravelmente de uma para outra.

$\mathrm{O}$ teor de $\mathrm{N}$ na planta apresentou diferenças significativas nos grãos e no teor total. Ademais, os tratamentos não influíram no teor de $\mathrm{N}$ da parte aérea, observando-se, ainda, que, diante da sua mobilidade, grande parte do elemento foi translocado para os grãos, onde os valores foram, praticamente, duas vezes maiores (Tabela 3).

Quanto ao Mo, observou-se que os efeitos dos tratamentos foram significativos entre as partes das plantas. Verificou-se, ainda, a eficiência da aplicação desse micronutriente via foliar, quando comparada com a testemunha e à peletização das sementes. Outro fator a considerar é a capacidade do FTE BR 9 no fornecimento do Mo, atuando de modo semelhante à adubação foliar com molibdato de amônio. 
TABELA 2. Teor foliar de nitrogênio e molibdênio no feijoeiro cultivar Goytacazes. Linhares, ES, $1995^{1}$.

\begin{tabular}{|c|c|c|c|c|c|}
\hline \multicolumn{4}{|c|}{ Tratamentos } & \multicolumn{2}{|c|}{ Teor foliar } \\
\hline \multicolumn{2}{|c|}{$\mathrm{N}(\mathrm{kg} / \mathrm{ha})$} & \multicolumn{2}{|c|}{ Mo (g/ha) } & \multirow{2}{*}{$\begin{array}{c}\mathrm{N} \\
(\mathrm{g} / \mathrm{kg})\end{array}$} & \multirow{2}{*}{$\begin{array}{c}\text { Mo } \\
(\mathrm{mg} / \mathrm{kg})\end{array}$} \\
\hline Plantio & Cobertura & Plantio & Cobertura & & \\
\hline 0 & 0 & 0 & 0 & $39,7 \mathrm{ab}$ & $0,23 \mathrm{f}$ \\
\hline 10 & 0 & 0 & 0 & $39,2 \mathrm{ab}$ & $0,27 \mathrm{ef}$ \\
\hline 0 & 40 & 0 & 0 & $40,1 \mathrm{ab}$ & $0,30 \mathrm{ef}$ \\
\hline 10 & 40 & 0 & 0 & $38,4 \mathrm{abc}$ & $0,37 \mathrm{ef}$ \\
\hline 0 & 0 & 0 & 20 & $36,4 b c$ & $1,17 b c$ \\
\hline 10 & 0 & 0 & 20 & $41,9 \mathrm{a}$ & $1,07 \mathrm{bc}$ \\
\hline 0 & 40 & 0 & 20 & $40,1 \mathrm{ab}$ & $1,67 \mathrm{a}$ \\
\hline 10 & 40 & 0 & 20 & $41,8 \mathrm{a}$ & $1,27 \mathrm{ab}$ \\
\hline 10 & 0 & 20 & 0 & $41,8 \mathrm{a}$ & $0,30 \mathrm{ef}$ \\
\hline 0 & 40 & 20 & 0 & $39,6 a b$ & $0,60 \mathrm{def}$ \\
\hline 10 & 40 & 20 & 0 & $37,8 \mathrm{abc}$ & $0,73 \mathrm{cde}$ \\
\hline 0 & 0 & 20 & 0 & $35,5 b c$ & $0,20 \mathrm{f}$ \\
\hline 10 & 40 & $40^{2}$ & 0 & $33,9 \mathrm{c}$ & $0,83 \mathrm{bcd}$ \\
\hline C.V. (\%) & & & & 6,64 & 36,83 \\
\hline
\end{tabular}

${ }^{1}$ Médias seguidas da mesma letra nas colunas não diferem entre si (Duncan 5\%).

2 Tratamento adicional: $40 \mathrm{~kg} / \mathrm{ha}$ de FTE BR 9 aplicado no sulco de plantio.

TABELA 3. Teor de nitrogênio e molibdênio na parte aérea e grãos do feijoeiro cultivar Goytacazes na época da colheita. Linhares, ES, 1995¹.

\begin{tabular}{|c|c|c|c|c|c|c|c|c|c|}
\hline \multicolumn{4}{|c|}{ Tratamentos } & \multicolumn{3}{|c|}{$\mathrm{N}(\mathrm{kg} / \mathrm{ha})$} & \multicolumn{3}{|c|}{ Mo (g/ha) } \\
\hline \multicolumn{2}{|c|}{$\mathrm{N}(\mathrm{kg} / \mathrm{ha})$} & \multicolumn{2}{|c|}{ Mo (g/ha) } & \multirow[t]{2}{*}{ P. aérea } & \multirow[t]{2}{*}{ Grão } & \multirow[t]{2}{*}{ Total } & \multirow[t]{2}{*}{ P.aérea } & \multirow[t]{2}{*}{ Grão } & \multirow[t]{2}{*}{ Total } \\
\hline Plantio & Cobertura & Plantio & Cobertura & & & & & & \\
\hline 0 & 0 & 0 & 0 & $40,55 \mathrm{a}$ & $79,96 \mathrm{abcd}$ & $120,52 \mathrm{a}$ & $2,82 \mathrm{ab}$ & $0,70 \mathrm{f}$ & $3,52 \mathrm{~d}$ \\
\hline 10 & 0 & 0 & 0 & $32,00 \mathrm{a}$ & 76,39abcd & $108,40 \mathrm{ab}$ & $2,25 \mathrm{ab}$ & $1,12 \mathrm{ef}$ & $3,37 d$ \\
\hline 0 & 40 & 0 & 0 & $34,55 \mathrm{a}$ & $85,73 \mathrm{ab}$ & $120,29 \mathrm{a}$ & 2,49ab & $0,79 \mathrm{f}$ & $3,29 \mathrm{~d}$ \\
\hline 10 & 40 & 0 & 0 & $35,67 \mathrm{a}$ & $80,24 \mathrm{abcd}$ & $115,91 \mathrm{ab}$ & $2,61 \mathrm{ab}$ & $1,83 \mathrm{ef}$ & $4,44 \mathrm{~cd}$ \\
\hline 0 & 0 & 0 & 20 & $35,16 a$ & $91,85 \mathrm{a}$ & $127,02 \mathrm{a}$ & $3,04 \mathrm{ab}$ & $8,11 \mathrm{a}$ & $11,15 \mathrm{a}$ \\
\hline 10 & 0 & 0 & 20 & $36,23 a$ & $82,80 \mathrm{abc}$ & $119,04 \mathrm{a}$ & $3,42 \mathrm{a}$ & $6,46 \mathrm{ab}$ & $9,88 \mathrm{ab}$ \\
\hline 0 & 40 & 0 & 20 & $33,95 a$ & $82,43 a b c$ & $116,40 \mathrm{ab}$ & $2,54 \mathrm{ab}$ & $6,01 \mathrm{abc}$ & $8,56 \mathrm{ab}$ \\
\hline 10 & 40 & 0 & 20 & $34,05 \mathrm{a}$ & $86,81 \mathrm{ab}$ & $120,86 \mathrm{a}$ & $2,13 \mathrm{ab}$ & $6,48 \mathrm{ab}$ & $8,61 \mathrm{ab}$ \\
\hline 10 & 0 & 20 & 0 & $35,38 \mathrm{a}$ & $82,06 \mathrm{abc}$ & $117,46 a$ & $2,34 \mathrm{ab}$ & 4,63bcd & $6,98 \mathrm{bc}$ \\
\hline 0 & 40 & 20 & 0 & $29,07 \mathrm{a}$ & $59,48 \mathrm{~d}$ & $88,56 \mathrm{~b}$ & $1,81 \mathrm{~b}$ & $2,56 \mathrm{def}$ & $4,38 \mathrm{~cd}$ \\
\hline 10 & 40 & 20 & 0 & $35,81 \mathrm{a}$ & $62,68 \mathrm{~cd}$ & $98,49 \mathrm{ab}$ & $2,56 a b$ & $2,35 \mathrm{def}$ & $4,92 \mathrm{~cd}$ \\
\hline 0 & 0 & 20 & 0 & $33,87 \mathrm{a}$ & $65,00 \mathrm{bcd}$ & $98,88 \mathrm{ab}$ & $1,83 \mathrm{~b}$ & $3,61 \mathrm{cde}$ & $5,45 \mathrm{~cd}$ \\
\hline 10 & 40 & $40^{2}$ & 0 & $39,72 \mathrm{a}$ & $75,25 \mathrm{abcd}$ & $114,98 \mathrm{ab}$ & $2,71 \mathrm{ab}$ & $6,44 \mathrm{ab}$ & $9,15 \mathrm{ab}$ \\
\hline C.V. (\%) & & & & 18,75 & 15,07 & 13,14 & 31,16 & 34,96 & 26,11 \\
\hline
\end{tabular}

${ }^{1}$ Médias seguidas da mesma letra nas colunas não diferem entre si (Duncan 5\%).

2 Tratamento adicional: $40 \mathrm{~kg} / \mathrm{ha}$ de FTE BR 9 aplicado no sulco de plantio. 
O tratamento mais produtivo proporcionou um incremento, na produção de grãos, de $108,7 \mathrm{~kg} / \mathrm{ha}$ em relação ao tratamento que não recebeu adubação (Tabela 1). Considerando os preços atuais do produto e dos insumos utilizados (uréia e molibdato de amônio), isto representa, economicamente, um ganho de $\mathrm{R} \$ 65,88 / \mathrm{ha}$.

\section{CONCLUSÕES}

1. Dentre os tratamentos estudados, a aplicação foliar de Mo e a adubação com FTE BR 9 no plantio são os que apresentam maior eficiência no fornecimento do elemento às plantas, superando a peletização das sementes.

2. A aplicação de $10 \mathrm{~kg} / \mathrm{ha}$ de $\mathrm{N}$ no plantio, $40 \mathrm{~kg} / \mathrm{ha}$ de $\mathrm{N}$ em cobertura e $20 \mathrm{~g} / \mathrm{ha}$ de Mo via foliar proporciona a maior produtividade de grãos.

\section{REFERÊNCIAS}

AMANE, M.I.V.; VIEIRA, C.; CARDOSO, A.A.; ARAÚJO, G.A.A. Resposta de cultivares de feijão (Phaseolus vulgaris L.) às adubações nitrogenadas e molíbdica. Revista Ceres, Viçosa, v.41, n.243, p.202-216, mar./abr. 1994.

BERGER, P.G.; VIEIRA, C.; ARAÚJO, G.A.A.; CASSINI, S.T.A. Peletização de sementes de feijão (Phaseolus vulgaris L.) com carbonato de cálcio, rizóbio e molibdênio. Revista Ceres, Viçosa, v.42, n.243, p.562-574, set./out. 1995.

BRAGA, J.M. Resposta do feijoeiro "Rico 23" à aplicação de enxofre, boro e molibdênio. Revista Ceres, Viçosa, v.19, n.103, p.222-226, maio/jun. 1972.

DEFELIPO, B.V.; RIBEIRO, A.C. Análise química do solo: metodologia. Viçosa: UFV, 1981. 17p. (Boletim de Extensão, 29).

EMBRAPA. Serviço Nacional de Levantamento e Conservação de Solos (Rio de Janeiro, RJ.). Levantamento de reconhecimento dos solos do
Estado do Espírito Santo. Rio de Janeiro, 1978. 461p. (Embrapa-SNLCS. Boletim técnico, 45).

EMBRAPA. Serviço Nacional de Levantamento e Conservação de Solos (Rio de Janeiro, RJ.). Manual de métodos de análise do solo. Rio de Janeiro, 1979. Não paginado.

JUNQUEIRA NETO, A.; SANTOS, O.S.; AIDAR, H.; VIEIRA, C. Ensaios preliminares sobre a aplicação de molibdênio e de cobalto na cultura do feijão (Phaseolus vulgaris L.). Revista Ceres, Viçosa, v.24, n.136, p.628-633, nov./dez. 1977.

MALAVOLTA, E.; VITTI, G.C.; OLIVEIRA, S.A. Avaliação do estado nutricional das plantas: princípios e aplicações. Piracicaba: POTAFOS, 1989. 201p.

MERCANTE, F.M.; STRALIOTTO, R.; DUQUE, F.F.; FRANCO, A.A. A inoculação do feijoeiro comum com rizóbio. Seropédica: Embrapa-CNPBS, 1992. 8p. (Embrapa-CNPBS. Comunicado técnico, 10).

SANTOS, A.B.; VIEIRA, C.; LOURES, E.G.; BRAGA, J.M.; THIÈBAUT, J.T.L. Resposta do feijoeiro (Phaseolus vulgaris L.) ao molibdênio e ao cobalto em solos de Viçosa e Paula Cândido, Minas Gerais. Revista Ceres, Viçosa, v.26, n.143, p.92-101, jan./fev. 1979.

SIQUEIRA, J.O.; FRANCO, A.A. Biotecnologia do solo: fundamentos e perspectivas. São Paulo: Nagy, 1988. 236p.

VIDOR, C.; KOLLING, J.; FREIRE, J.R.J.; SCHOLLES, D.; BROSE, E.; PEDROSO, M.H.T. Fixação biológica de nitrogênio pela simbiose entre Rhizobium e leguminosas. Porto Alegre: IPAGRO, 1983. 51p. (IPAGRO. Boletim técnico, 11).

VIEIRA, C. Cultura do feijão. Viçosa: UFV, 1983. 146p.

VIEIRA, C.; NOGUEIRA, A.O.; ARAÚJO, G.A.A. Adubação nitrogenada e molíbdica na cultura do feijão. Revista de Agricultura, Piracicaba, v.67, n.2, p.117-124, 1992.

VIEIRA, R.F. Aplicação foliar de molibdênio e seu efeito nas atividades da nitrogenase e redutase do nitrato do feijoeiro em campo. Piracicaba: ESALQ, 1994. 188p. Tese de Doutorado. 